\title{
PROCESAMIENTO DE IMÁGENES EN LA CARACTERIZACIÓN DE UNA TORCHA HIDRÁULICA
}

\section{IMAGE PROCESSING IN VORTEX ROPE CHARACTERIZATION}

\author{
MSc Hernan Bolaños, MSc Guillermo Angarita, Msc Sylvain Richard, PhD \\ Francisco Botero
}

Universidad Eafit, Escuela de Ingeniería, Departamento de Ingeniería Mecánica Cr 49 No 7 sur 50, Medellín, Antioquia, Colombia Teléfono: (+57 4) 2619500 ext. 9827

E-mail: hbolanos@eafit.edu.co, langari3@eafit.edu.co, srichard@chakipka.ch, fboteroh@eafit.edu.co

\begin{abstract}
Resumen: Turbinas Kaplan o Francis trabajando en condiciones fuera de diseño pueden exhibir fenómenos hidrodinámicos como la torcha, que representan riesgos para la instalación y operación de turbinas. En el presente estudio se reprodujo este tipo de fenómeno en una bomba centrífuga trabajando como turbina.

Para caracterizar el fenómeno, los movimientos periódicos de la torcha fueron estimados por dos métodos comparables entre sí. El primero, procesamiento de imágenes de una secuencia de fotos tomada con una cámara de alta velocidad, y el segundo, señales de sensores de presión consideradas como fluctuaciones de presión.

Análisis en el dominio del tiempo y la frecuencia permitieron encontrar la relación entre espectros de la torcha y las áreas proyectadas en la secuencia de imágenes del fenómeno.
\end{abstract}

Palabras clave: Torcha hidráulica, procesamiento de imágenes, espectros, fenómenos hidrodinámicos

\begin{abstract}
Kaplan or Francis turbines working at off-design conditions can exhibit hydrodynamic phenomena such as vortex rope, which can affect the turbines installation and operation. In this study, vortex rope was reproduced in a pump as turbine. In this case, periodic movements in vortex rope were elliptical and straight on the flow sense.

To characterize the phenomenon of interest, the periodic movements of vortex rope were estimated by two methods comparing each other. First, image processing of sequence of pictures taken by a high-speed camera, and second, pressure sensors signals regarded such as pressure fluctuations.

The time and frequency domain analysis allowed to find out the relationship between vortex rope spectral components and projected areas in image sequence of vortex rope.
\end{abstract}

Keywords: Vortex rope, image processing, spectra, hydrodynamic phenomena.

\section{INTRODUCCIÓN}

Muchas zonas rurales en Colombia no están conectadas al sistema eléctrico nacional, por lo cual la generación de energía eléctrica se convierte en una necesidad y en un reto en estos lugares. Una propuesta para resolver este problema ha sido el diseño y construcción de microturbinas Pelton (Bohórquez, 2018), y otra el uso de bombas como turbinas (PAT, Pump as Turbine, por sus siglas en inglés). Esta segunda propuesta implica la identificación y caracterización de fenómenos hidrodinámicos como la torcha hidrúalica que 
afectan el desempeño y la eficiencia de las turbomáquinas.

En turbinas tipo Francis, la torcha se caracteriza típicamente por tener una forma helicoidal y por presentar dos tipos de movimientos periódicos, uno debido a la traslación y otro debido a la rotación sobre su propio eje (Nicolet et al, 2010). El movimiento de traslación es el que caracteriza la frecuencia de la torcha e induce fluctuaciones de presión convectivas locales en el tubo de aspiración, las cuales se propagan a todo el sistema hidráulico (Favrel et al, 2014). La propagación de las fluctuaciones de presión se da a la frecuencia de traslación de la torcha (Favrel et al, 2015). Adicionalmente, la presencia de torcha es una muestra de la disminución del nivel de presión en el cono de aspiración y por consiguiente de la aparición de cavitación (Favrel et al, 2015). Lo anterior, unido a que el fenómeno se presenta en condiciones de carga parcial de caudal, puede poner en riesgo la estabilidad de las turbinas.

Dado que las bombas centrífugas pueden invertir el giro de rotación del impulsor, se pueden operar como turbinas. De allí que resulte de interés replicar y caracterizar aquellos fenómenos hidrodinámicos que afectan a las turbinas, y observar de qué manera tienen su correspondencia en las bombas operadas como turbinas. Por lo anterior, se definió como objeto principal de esta investigación la caracterización de la torcha hidráulica en una bomba operada como turbina utilizando dos métodos comparables entre sí, procesamiento de imágenes y análisis de fluctuaciones de presión.

\section{MÉTODOS}

\subsection{Banco de pruebas}

El banco de pruebas está conformado por un circuito cerrado de tuberías y un banco de bombas centrifugas que suministra la cabeza hidráulica necesaria para el desarrollo de los experimentos. En el circuito está integrada la bomba centrífuga objeto de estudio que corresponde a una ITT Goulds de 1.5 HP de potencia nominal. La bomba cuenta con tuberías trasparentes para la visualización de los fenómenos hidrodinámicos en las zonas de alta y baja presión.

\subsection{Instrumentación y adquisición de datos}

Sensores de presión piezorresistivos Wika A10 fueron colocados alrededor de los orificios de baja y alta presión de la bomba. Sobre la voluta, y con una separación de $30^{\circ}$, se colocaron un sensor piezorresisitivo Futek PMP300 y un piezoeléctrico Dytran 2005V. La instrumentación fue complementada con un medidor de flujo ultrasónico GE TransPort PT878, que se instaló aguas arriba de la bomba como turbina, con un sensor de torque Futek TRS600 FSH01998, instalado en el eje de la bomba, y con un tacómetro DT2234C+, que registró la velocidad de la bomba en su eje.

Las señales de todos los sensores fueron registradas por un Equipo de Adquisición de Datos (EAD) CompactRio® 9076 de National Instruments ${ }^{\circledR}$ y un software llamado Turbologger. El EAD se configuró con cuatro módulos, dos NI-9232, un NI9215 y un NI-9203. El software Turbologger fue desarrollado en ambiente LabView® por investigadores de la Universidad Eafit.

El registro de todas las señales fue realizado simultáneamente, con una frecuencia de muestreo de $20480 \mathrm{~Hz}$ durante $32 \mathrm{~s}$. La frecuencia máxima observable fue de $10240 \mathrm{~Hz}$ con una resolución de $0.03125 \mathrm{~Hz}$.

\subsection{Procesamiento de imágenes}

Dada la configuración del experimento, el tubo de baja presión cumplió la función de tubo de aspiración de la bomba como turbina y, a través de éste, se tomaron las imágenes con una cámara de alta velocidad Photron Fastcam SA4, para un punto de operación en la zona de turbina-freno. La velocidad de captura de las imágenes fue de 3000 imágenes por segundo, con una apertura de diafragma de 1/3000 s. Para el análisis se tomó una parte de la secuencia con una duración de $1.312 \mathrm{~s}$ (3935 fotos, desde la imagen 2501 a la 6435). La resolución de cada imagen fue de $1024 \times 512$ pixeles.

A cada imagen se le extrajo una parte que correspondió a una zona dentro del tubo de aspiración con la torcha incluida $\left(\mathrm{A}_{\mathrm{i}}\right)$, de $901 \times 229$ pixeles. Sobre cada imagen recortada se hizo un proceso de filtrado para determinar el área de la torcha $\left(\mathrm{A}_{\mathrm{t}}\right)$ dentro de la imagen. Las áreas fueron medidas en pixeles ${ }^{2}$. La relación $\mathrm{A}_{\mathrm{t}} / \mathrm{A}_{\mathrm{i}}$ se considera representativa del diámetro de la torcha (Nicolet et al, 2010). 


\subsection{Fluctuación de presiones}

Todas las presiones registradas fueron expresadas como coeficientes de fluctuaciones de presión, de acuerdo con lo definido en la norma CEI/IEC 60193 (IEC, 1999), de la siguiente manera:

$$
\widetilde{P}_{E}=\frac{p-\bar{p}}{\rho E}
$$

Donde,

$\widetilde{P}_{E}$ : coeficiente de fluctuación de presión [-]

$p$ : presión [Pa]

$\bar{p}$ : presión promedia $[\mathrm{Pa}]$

$\rho:$ densidad $\left[\mathrm{kg} / \mathrm{m}^{3}\right]$

$E$ : energía específica $[\mathrm{J} / \mathrm{kg}]$

\subsection{Análisis de datos}

Las series de relaciones de áreas de las imágenes y de presiones se analizaron en el dominio del tiempo para identificar patrones de comportamiento y en el dominio de la frecuencia para identificar componentes espectrales similares. El análisis espectral es una técnica ampliamente utilizada para identificar fallas en máquinas rotativas (Reyes et al, 2007), así como fenómenos hidrodinámicos como la torcha, el Rotating Stall o el Surge.

Las frecuencias se expresaron en términos de coeficientes de frecuencia, tal como lo señala la norma CEI/IEC 60193 (IEC, 1999):

$$
f_{n}=\frac{f}{n}
$$

Donde,

$f_{n}$ : coeficiente de frecuencia [-]

$f:$ frecuencia $\left[\mathrm{s}^{-1}\right]$

$n$ : frecuencia de rotación del rodete $\left[\mathrm{s}^{-1}\right]$

Otros números adimensionales utilizados en el presente trabajo como el factor de caudal, $\mathrm{Q}_{\mathrm{ED}}$, el factor de velocidad, $n_{E D}$, y el coeficiente de energía, $\mathrm{E}_{\mathrm{nD}}$, fueron definidos como lo establece la norma CEI/IEC 60193 (IEC, 1999) tal como se presenta a continuación:

$$
\begin{aligned}
& n_{E D}=\frac{n D}{E^{0.5}} \\
& Q_{E D}=\frac{Q}{D^{2} E^{0.5}} \\
& E_{n D}=\frac{E}{n^{2} D^{2}}
\end{aligned}
$$

Donde,

$D$ : Diámetro de referencia [m]

$Q$ : Caudal $\left[\mathrm{m}^{3} / \mathrm{s}\right]$

\section{RESULTADOS}

La curva característica $\mathrm{QED}_{\mathrm{ED}} \mathrm{n}_{\mathrm{ED}}$ de la bomba como turbina se presenta en la figura 1. Allí se puede observar el Punto de Máxima Eficiencia (PME), el punto de embalamiento y el Punto de Operación de Interés (POI). Este punto marca el inicio de la forma "s" en la zona de turbina-freno.

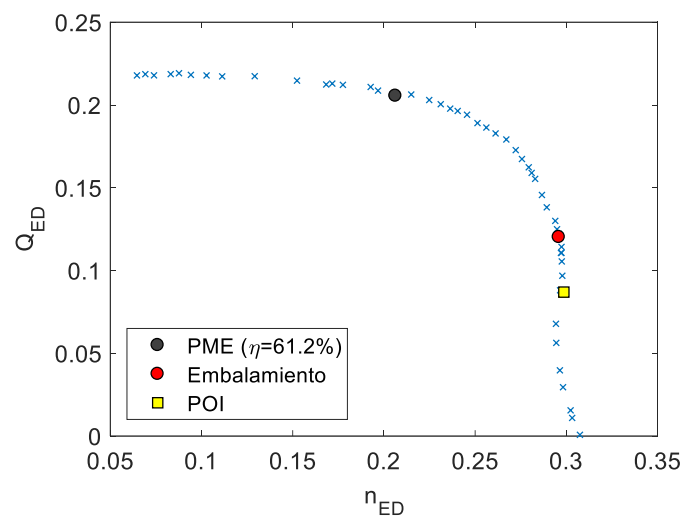

Fig 1. Curva característica $Q_{E D}-n_{E D}$ de la bomba como turbina

Parámetros del POI se muestran en la Tabla 1. En ésta se puede observar que la relación QED POI/QED PME corresponde a un punto de baja carga parcial. En la tabla 1 también se presenta la relación de coeficientes de energía entre el POI y el PME.

Tabla 1: Algunos parámetros del POI

\begin{tabular}{|c|c|c|c|c|}
\hline $\mathbf{n}\left[\mathbf{s}^{-1}\right]$ & QED & nED & $\begin{array}{c}\text { QED_POI/ } \\
\text { QED_PME }\end{array}$ & $\begin{array}{c}\text { EnD_POI }_{\text {En_PME }} \\
\text { En_P }^{-1}\end{array}$ \\
\hline 26.44 & 0.0870 & 0.2986 & 0.4232 & 0.4773 \\
\hline
\end{tabular}

\subsection{Procesamiento de imágenes}

La figura 2 muestra dos imágenes a modo de ejemplo con sus correspondientes partes sustraídas y filtradas, donde el área blanca corresponde al área de la proyección de la torcha sobre un plano vertical y la parte negra corresponde a su entorno.

La secuencia de imágenes considerada puso de manifiesto dos movimientos periódicos en la torcha: uno semihelicoidal y otro longitudinal de menor frecuencia que el primero. 


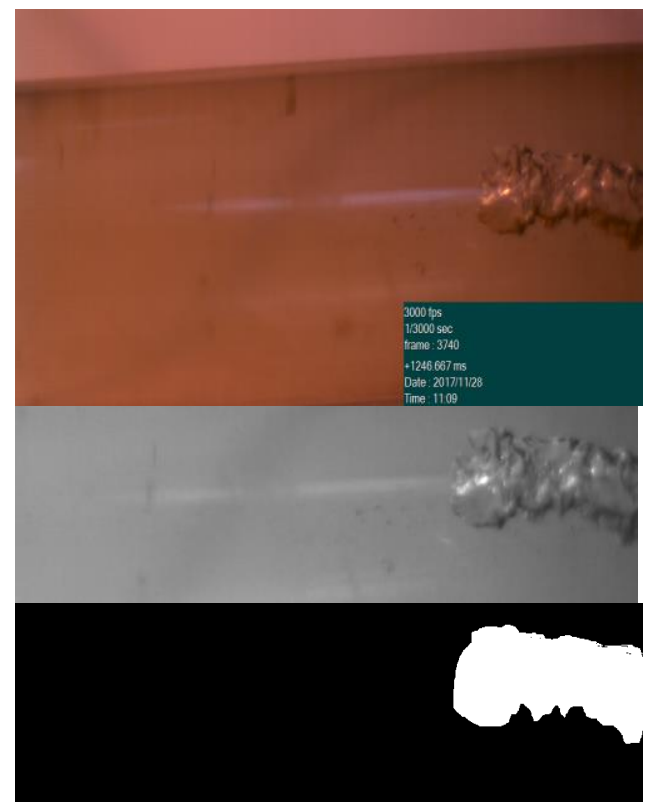

a)

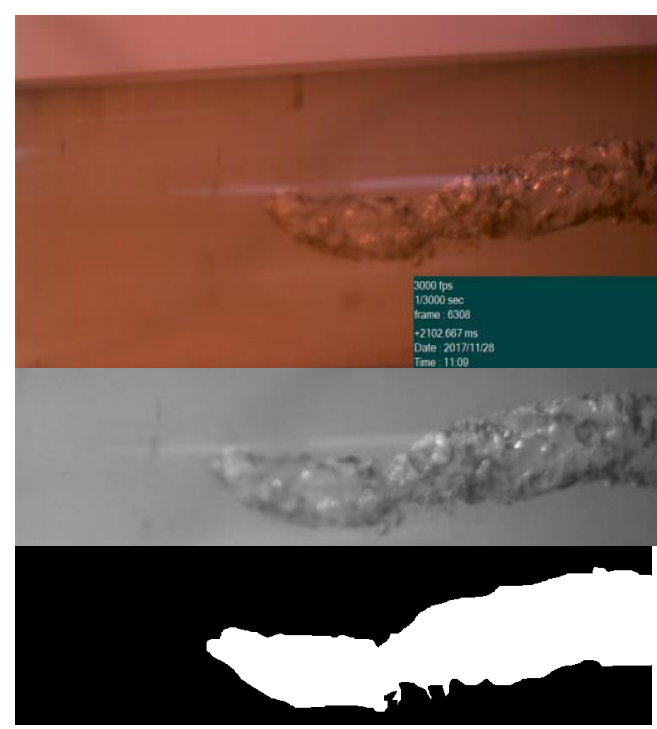

b)

Fig. 2. Procesamiento de imágenes, a) Imagen 3740 (1241 de la secuencia considerada) y b) Imagen 6308 (3809 de la secuencia considerada)

En la figura 3 se muestra la serie de relaciones de área en el domino del tiempo. Allí se pueden ver dos patrones claramente definidos, uno, que corresponde al periodo promedio para el movimiento semihelicoidal, $\mathrm{T}_{\mathrm{sh}}=0.0638 \mathrm{~s}$, y otro, al periodo promedio para el movimiento longitudinal, $\mathrm{T}_{\mathrm{l}}=0.5420 \mathrm{~s}$. Con estos periodos se estimó la frecuencia del movimiento semihelicoidal,
$\mathrm{F}_{\mathrm{sh}}=15.68 \mathrm{~Hz}, \mathrm{y}$ del movimiento longitudinal, $\mathrm{F}_{1}=1.85 \mathrm{~Hz}$.

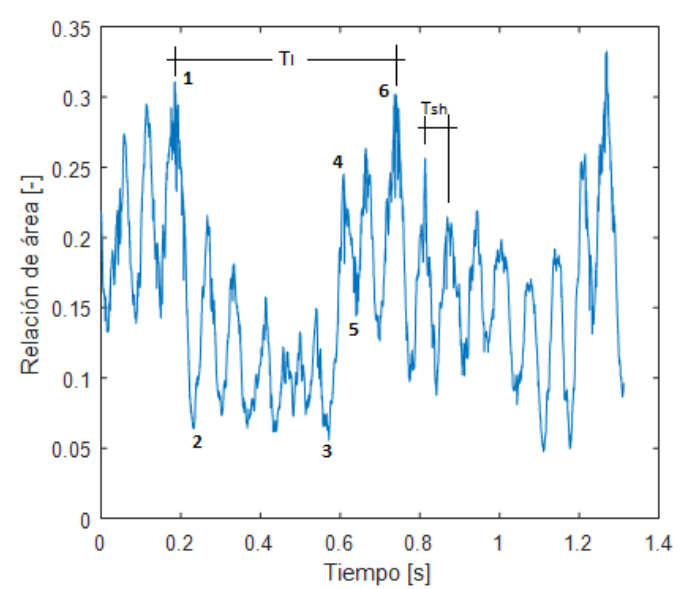

Fig 3. Serie de relaciones de área en el dominio del tiempo

Analizando en conjunto las imágenes con la gráfica de relación de áreas (Fig. 3) se observa que el patrón del movimiento helicoidal es constante a lo largo del tiempo, en tanto que el patrón del movimiento longitudinal presenta una serie de variaciones muy particulares que se entran a detallar a continuación:

- En la secuencia de 1 a 2, la torcha se desplaza horizontalmente el equivalente a su amplitud máxima $\left(\mathrm{a}_{1}\right)$, en un periodo de tiempo aproximado a $0.09 \mathrm{~T}_{1}$. En la secuencia de 2 a 6 se desplaza otra distancia equivalente $a a_{1}$ en un tiempo aproximado de $0.91 \mathrm{~T}_{1}$.

- En la secuencia de 2 a 3, la torcha se queda oscilando con una amplitud hasta de 0.6 al.

- En la secuencia de 4 a 5, la torcha se queda girando sobre su eje, pero con desplazamiento horizontal prácticamente nulo. Varía su relación de área, pero no hay movimiento horizontal significativo.

- En la secuencia de 5 a 6, la torcha oscila hasta alcanzar su máxima amplitud nuevamente.

En la figura 4 se presentan las imágenes correspondientes a los puntos 1 a 6 de la figura 3, donde el borde derecho de la imagen y la línea vertical blanca definen la amplitud máxima de la torcha en dirección horizontal. Cabe resaltar que la variación de la relación de áreas (Fig. 3) describe de una manera adecuada, entre otras cosas, el comportamiento de la amplitud de la torcha en dirección horizontal. 


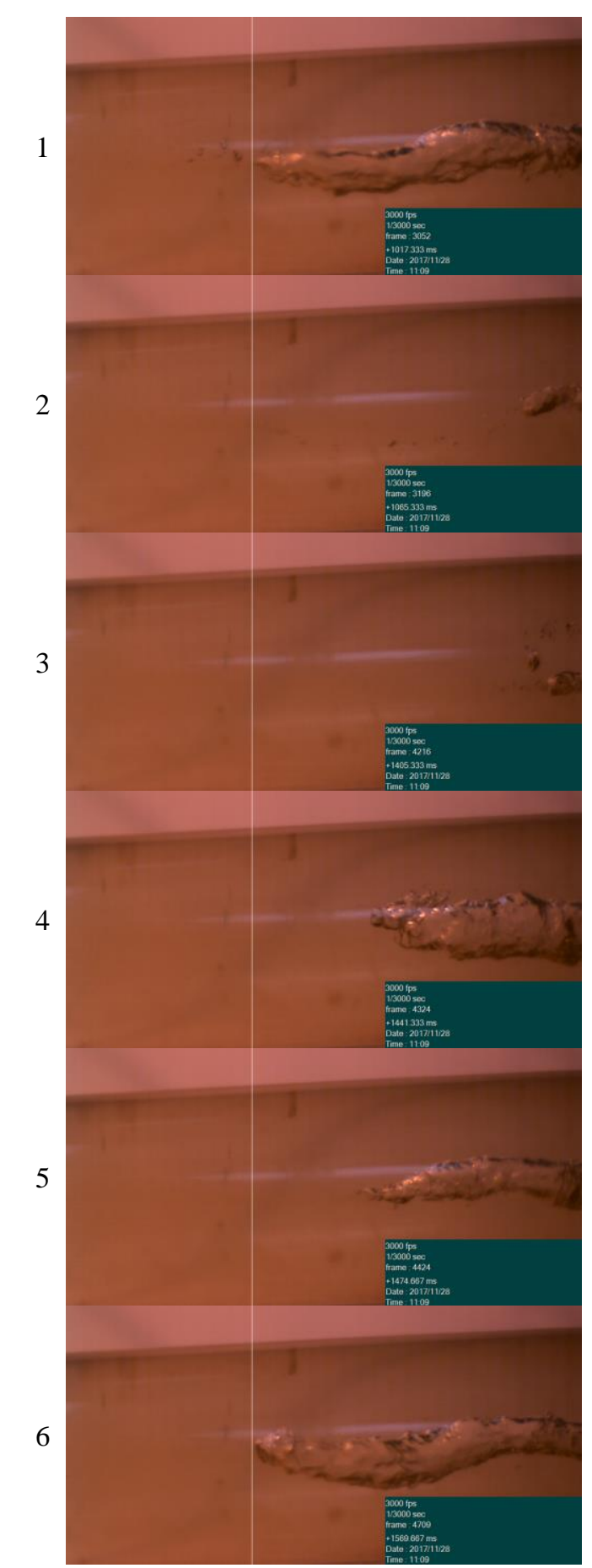

Fig 4. Torcha en un periodo promedio de $1 T_{l}$

\subsection{Señales de presión}

En la figura 5 se muestra el comportamiento de las fluctuaciones de presión de los sensores instalados en la bomba como turbina, en los puntos de operación de máxima eficiencia y de interés. En el PME no se notan perturbaciones significativas en las señales de presión de todos los sensores, en tanto que en el POI si es evidente la perturbación. La comparación entre las figuras $5 a$ ) y 5 b) pone de manifiesto la propagación de las fluctuaciones de presión a todo el sistema hidráulico, tal como lo mencionan (Nicolet et al, 2010) y (Favrel et al, 2014).

El análisis de las fluctuaciones de presión en el dominio de la frecuencia se realizó por medio de la función de densidad del espectro cruzado. Esto permitió identificar tres componentes a nivel subsincrónico en el POI. Dos de ellos sugieren una relación directa con las frecuencias identificadas en el análisis de imágenes, y el otro $\left(f_{n}=0.8303\right)$ sugiere como fuente un fenómeno diferente que está por fuera del alcance de este trabajo.

En la figura 6a) se presenta la magnitud de la función de densidad espectral cruzada para tres casos: primero, las fluctuaciones de presión a partir del Sensor PiezoEléctrico (SPE) instalado en la voluta con las fluctuaciones de presión de la señal del Sensor PiezoRresistivo (SPR) en la voluta; segundo, las fluctuaciones de presión del SPE montado en la voluta con el SPR en la zona de alta presión, y, tercero, las fluctuaciones de presión del SPE de la voluta con las del SPR en la zona de baja presión. Esta figura presenta dos componentes espectrales $\left(\mathrm{f}_{\mathrm{n}}=0.0561\right.$ y $\left.\mathrm{f}_{\mathrm{n}}=0.5585\right)$ que sugieren tener relación con las frecuencias de los dos movimientos de la torcha identificados mediante el análisis de imágenes. Adicionalmente, presenta otro componente espectral de interés en $\mathrm{f}_{\mathrm{n}}=0.1152$.

La figura 6b) indica las correspondientes coherencias para los componentes espectrales identificados en la figura 6a). Estos componentes presentan coherencias superiores al $96 \%$ para los componentes $\mathrm{f}_{\mathrm{n}}=0.5585$ y $\mathrm{f}_{\mathrm{n}}=0.1152$, y superiores al $86 \%$ para el caso del componente $\mathrm{f}_{\mathrm{n}}=0.0561$.

En la tabla 2 se muestra la comparación de las frecuencias halladas por los dos métodos en términos del factor de frecuencia estimado con respecto a la velocidad de rotación de la bomba como turbina. Los resultados muestran una buena aproximación en las frecuencias estimadas por los dos métodos para el caso del movimiento semihelicoidal (diferencia menor al 6\%), y aceptable para el caso del movimiento longitudinal dado que se trata de unas frecuencias muy pequeñas (diferencia del 20\%). 
Tabla 2: Frecuencias obtenidas por los dos métodos

\begin{tabular}{|l|r|c|c|c|}
\hline \multicolumn{1}{|c|}{ Método } & $\mathbf{F}_{\text {sh }}[\mathbf{H z}]$ & $\mathbf{f n}_{\text {sh }}$ & $\mathbf{F}_{\mathbf{I}}[\mathrm{Hz}]$ & $\mathbf{f n}_{\mathbf{I}}$ \\
\hline Imágenes & 15.68 & 0.5930 & $1.85 \mathrm{~Hz}$ & 0.0698 \\
\hline $\begin{array}{l}\text { Señales de } \\
\text { presión }\end{array}$ & 14.77 & 0.5585 & $1.48 \mathrm{~Hz}$ & 0.0561 \\
\hline
\end{tabular}
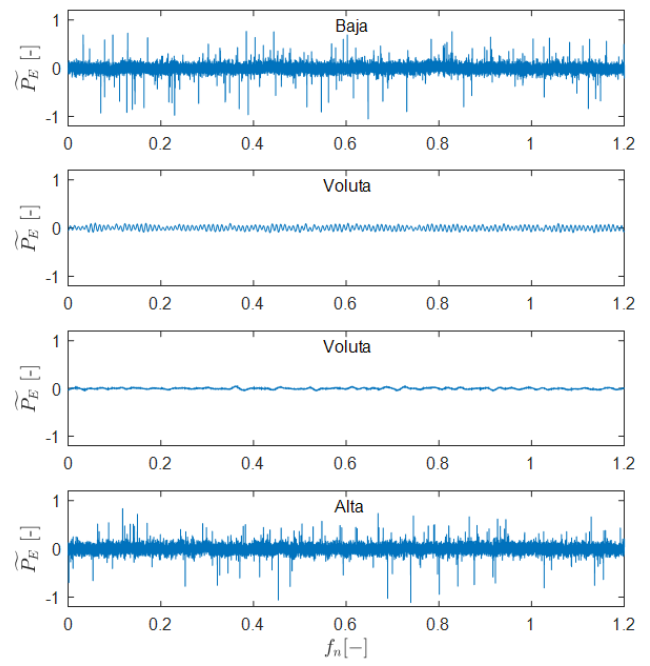

a)
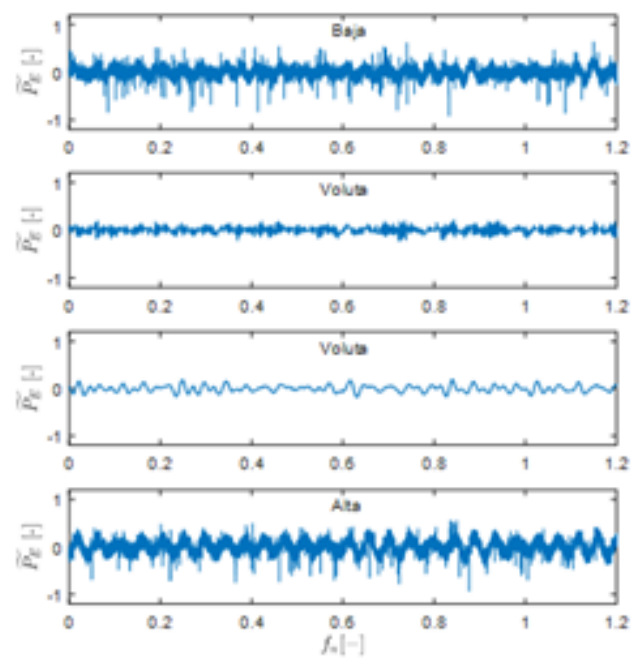

b)

Fig 5. Fluctuaciones de presiones en el PME (a) y en el POI $(b)$

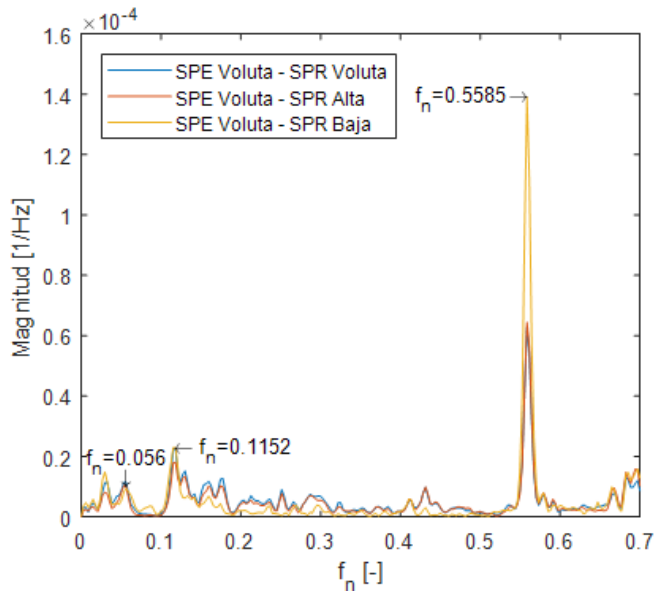

a)

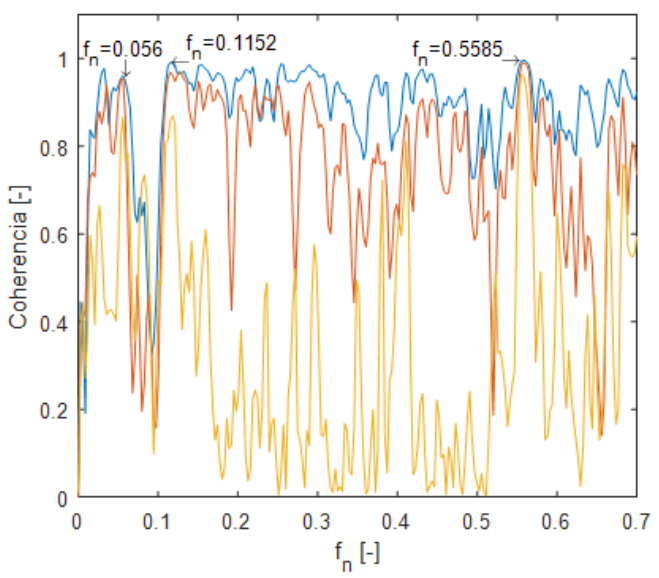

b)

Fig 6. a) Magnitud de la función de densidad del espectro cruzado y b) coherencia de los componentes espectrales de la misma función

Para complementar el análisis anterior, se realizó un espectro de frecuencias para la relación de áreas (véase Figura 7). Allí se muestran tres componentes espectrales que están en el mismo orden de magnitud de los componentes espectrales identificados por medio de la función de densidad del espectro cruzado. Lo anterior sugiere que, primero, los dos métodos están identificando las mismas frecuencias asociadas a la torcha, y, segundo, que hay un tercer movimiento periódico asociado al fenómeno hidrodinámico. El tercer movimiento podría determinar el movimiento particular que se da en sentido longitudinal y que resulta bastante interesante ya que el desplazamiento de la torcha hasta su amplitud máxima en un sentido se realiza en un tiempo mucho menor que en el otro sentido. Sin embargo, 
y al no tener más evidencias, no se puede comprobar esta hipótesis.



Fig 7. Espectro de frecuencias para la relación de áreas

\section{CONCLUSIONES}

A pesar de que el tubo de aspiración no contó con sensores de presión, los sensores instalados en otras partes de la turbomáquina permitieron identificar el efecto del fenómeno de interés sobre el sistema hidráulico.

Si bien no se puede hacer una comparación directa entre la torcha observada en este trabajo con las que se pueden presentar en las turbinas tipo Francis, es claro que el fenómeno está gobernado principalmente, en ambos casos, por dos movimientos periódicos que se pueden notar en el procesamiento de imágenes y en el análisis se señales de presión. En el caso de este estudio, el movimiento semihelicoidal sería equivalente al giro de la torcha sobre su propio eje que mencionan (Nicolet et al, 2010), y el movimiento longitudinal correspondería al de traslación.

La evidencia recogida sugiere que los dos métodos son comparables, en este caso de estudio en particular, para describir los movimientos de la torcha sobre su propio eje y en sentido longitudinal, aclarando que la diferencia entre los estimativos de las frecuencias es menor para el movimiento sobre el propio eje que para el movimiento longitudinal.

La evidencia recogida sugiere la existencia de un tercer movimiento de la torcha que se puede identificar a través de la comparación de los espectros de la serie de relación de áreas y la función de densidad del espectro cruzado de las señales de presión.

\section{RECONOCIMIENTO}

Los autores quieren expresar su gratitud a la Dirección de Investigación, el laboratorio de Hidráulica, el laboratorio de Metrología y el laboratorio de Mecatrónica de la universidad EAFIT por todo el soporte técnico y logístico brindado para el desarrollo de esta investigación.

\section{REFERENCIAS}

Bohórquez, A. (2018). "Microturbina Peltón, una solución real de energía para zonas no interconectadas (ZNI)". Revista Colombiana de Tecnologías de Avanzada, Vol. 1, No. 31. doi: 10.24054/16927257.v31.n31.2018.2768

C. J. Torres, J. F. Archila, M. L. Tronco, M. Becker, A. J. V. Porto, A. J. Tiberti. (2013). Estudio cinemático de una plataforma robótica para agricultura. REVISTA COLOMBIANA DE TECNOLOGÍAS DE AVANZADA, ISSN: 1692-7257. 2(22).

Nicolet, C., Zobeiri, A., Maruzewski, P., Avellan, F. (2010). "On the upper part load vortex rope in Francis turbine: experimental investigation". IOP Conf. Series: Earth and Environmental Science, Vol. 12, No. 012053. 1315/12/1/012053

doi:10.1088/1755-

Favrel, A., Landry, C., Muller, A., Yamamoto, K. Avellan, F. (2014). "Hydro-acoustic resonance behavior in presence of a precessing vortex rope: observation of a lockin phenomenon at part load Francis turbine operation". IOP Conf. Series: Earth and Environmental Science, Vol. 22, No. $032035 . \quad$ doi:10.1088/1755$1315 / 22 / 3 / 032035$

Favrel, A., Landry, C., Muller, A., Yamamoto, K. Avellan, F. (2015). "Study of the vortexinduced pressure excitation source in a Francis turbine draft tube by particle image velocimetry”. Exp Fluids, Vol. 56, No. 215. doi: $10.1007 / \mathrm{s} 00348-015-2085-5$

International Electrotechnical Commission (1999). "CEI/IEC 60193. Hydraulic turbines, storage pumps and pump-turbines, Model acceptance tests". Switzerland.

Reyes, F., Alvarado, R., Aranguren, S. (2007). "Sistema de detección de fallas en máquinas 
rotativas industriales basado en el método de análisis espectral". Revista Colombiana de Tecnologías de Avanzada, Vol. 2, No. 10, pp 64-72.

Torres, C., Archila, J., Tronco, M., Becker, M., Viera Porto, A., \& Tiberti, A. (2013). Estudio cinemático de una plataforma robótica para agricultura. REVISTA COLOMBIANA DE TECNOLOGÍAS DE AVANZADA, 2(22). 